\title{
EDITORIAL
}

Koji Okamoto · Jun Sakakibara

\section{The 16th international symposium on flow visualization, ISFV16}

Published online: 27 June 2015

(c) The Visualization Society of Japan 2015

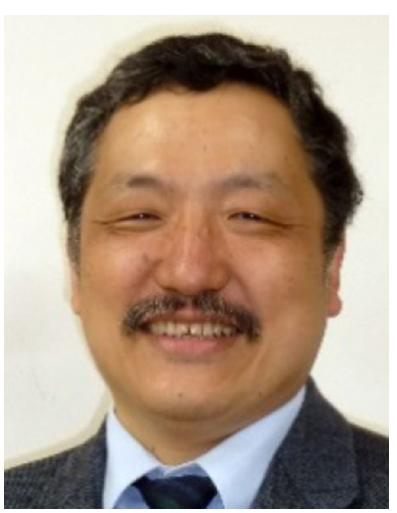

Koji Okamoto, Chair of ISFV16

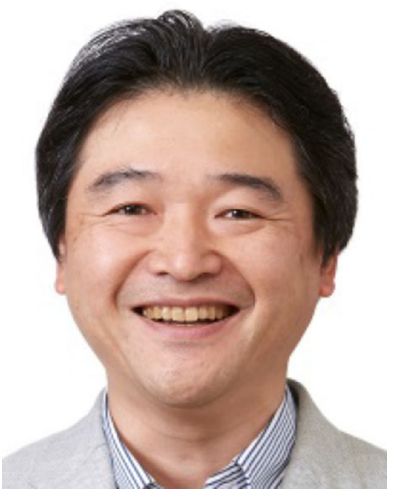

Jun Sakakibara, Technical Committee Chair of ISFV16

The 16th International Symposium on Flow Visualization (ISFV16) was held at Okinawa Convention Center, Okinawa, Japan, on 24-27 June 2014. ISFV16 was hosted by the Visualization Society of Japan, the official society of Journal of Visualization. This special issue contains the best papers selected from ISFV16. At ISFV16, the state of arts in many aspects of flow visualization was presented and discussed, including 3 special lectures, 6 invited lectures and over 250 technical papers.

The international symposium on flow visualization started in 1977 in Tokyo. The Visualization technique for flow was just the photographs and so on. After 40 years of progress, many techniques have been developed and applied to understand the flow. For example, particle image velocimetry (PIV) is now the standard tool to understand the flow characteristics. This special issue will help the progress of visualization, and will be a milestone to the future of flow visualization.

Okinawa is the beautiful southern island of Japan. Many participants around the world enjoyed the Okinawa's early summer. The Ryukyu hospitality was also welcomed. The Ryukyu kingdom was an old Okinawa kingdom.

Next international symposium on flow visualization will be held at Smokey Mountain, United States in 2016.

K. Okamoto $(\square)$

The University of Tokyo, Tokyo, Japan

E-mail: okamoto@n.t.u-tokyo.ac.jp

J. Sakakibara

Meiji University, Tokyo, Japan 